Figure 1A
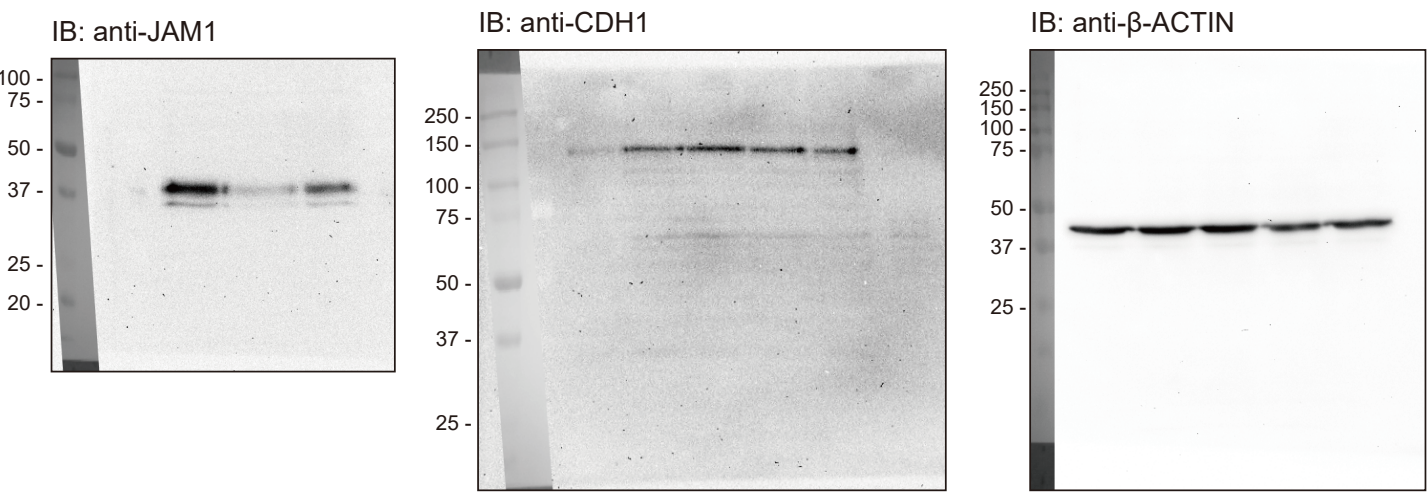

IB: anti-DSC2

IB: anti-NECTIN1
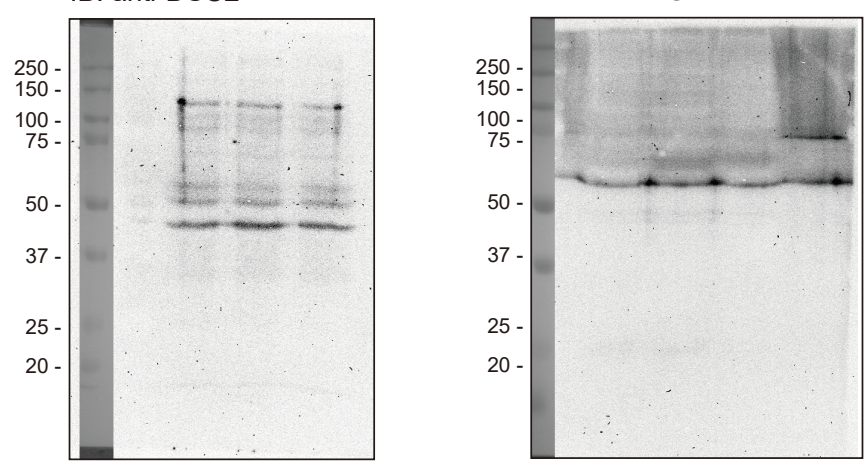

Figure 1B

IB: anti-Myc
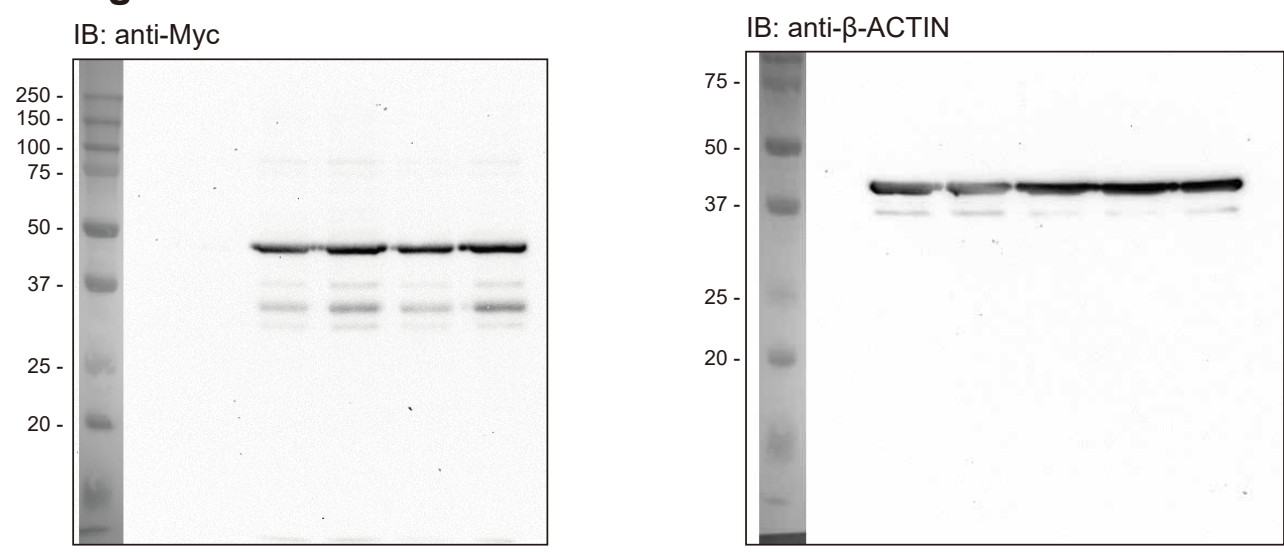

\title{
Figure 1C
}

IB: anti-Myc

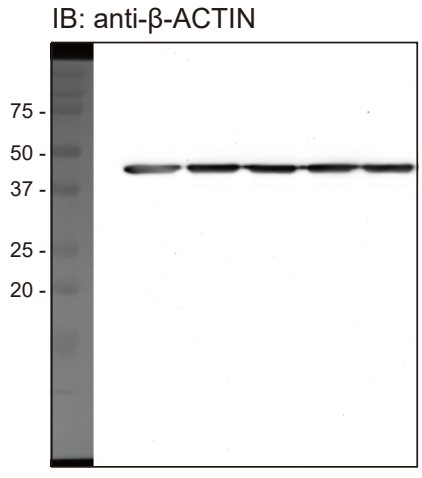


Figure 1D
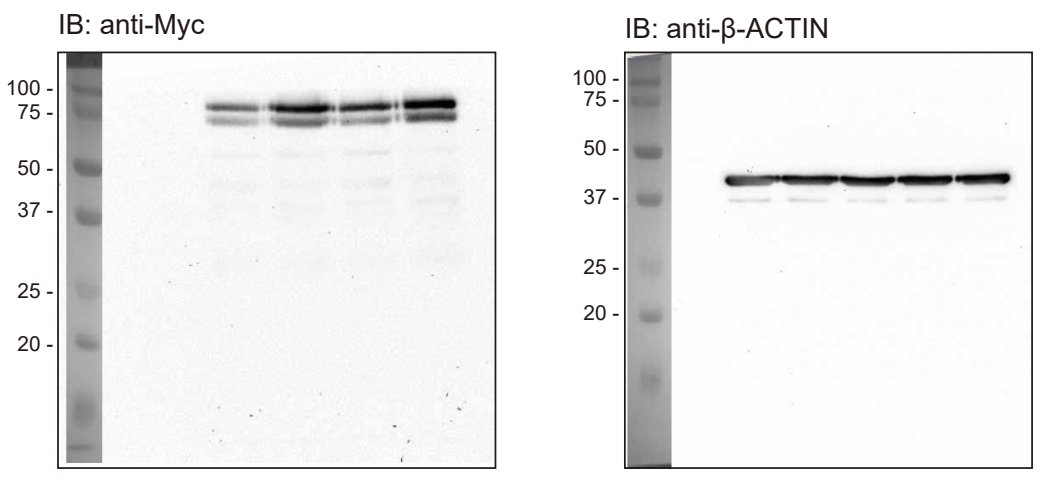

\section{Figure 1E}
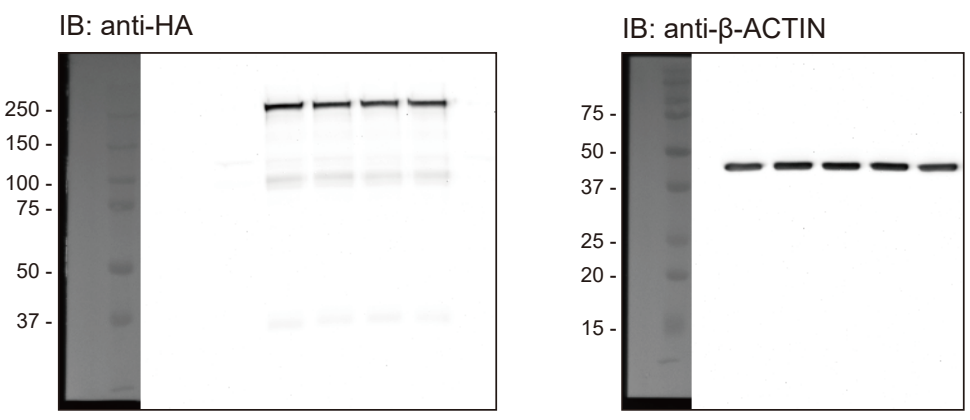

Figure 4B

IB: anti-HA
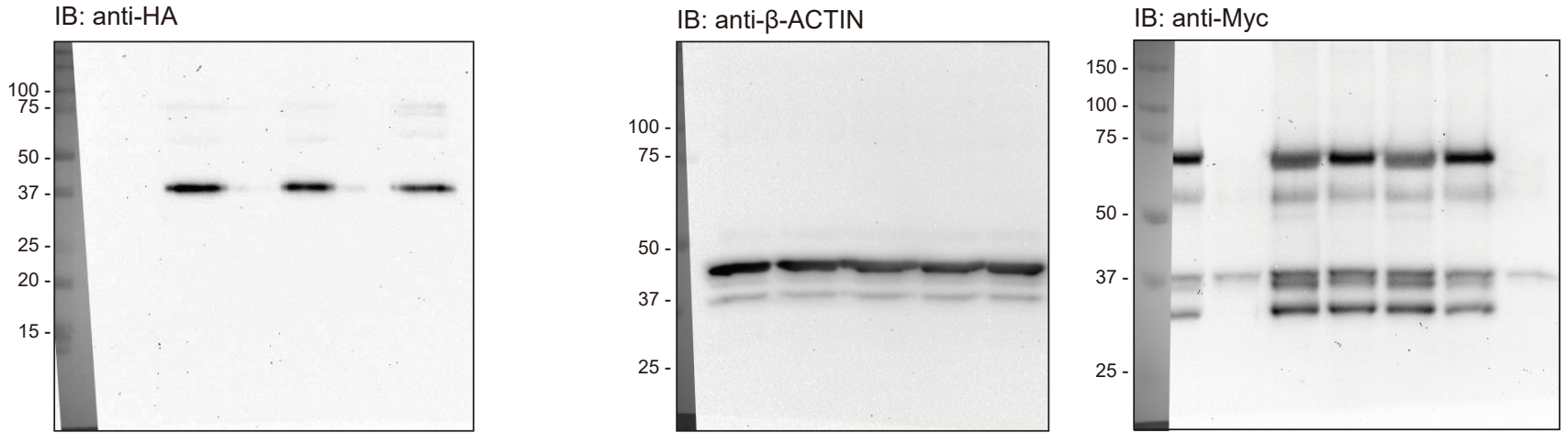

Figure 4C

IB: anti-HA
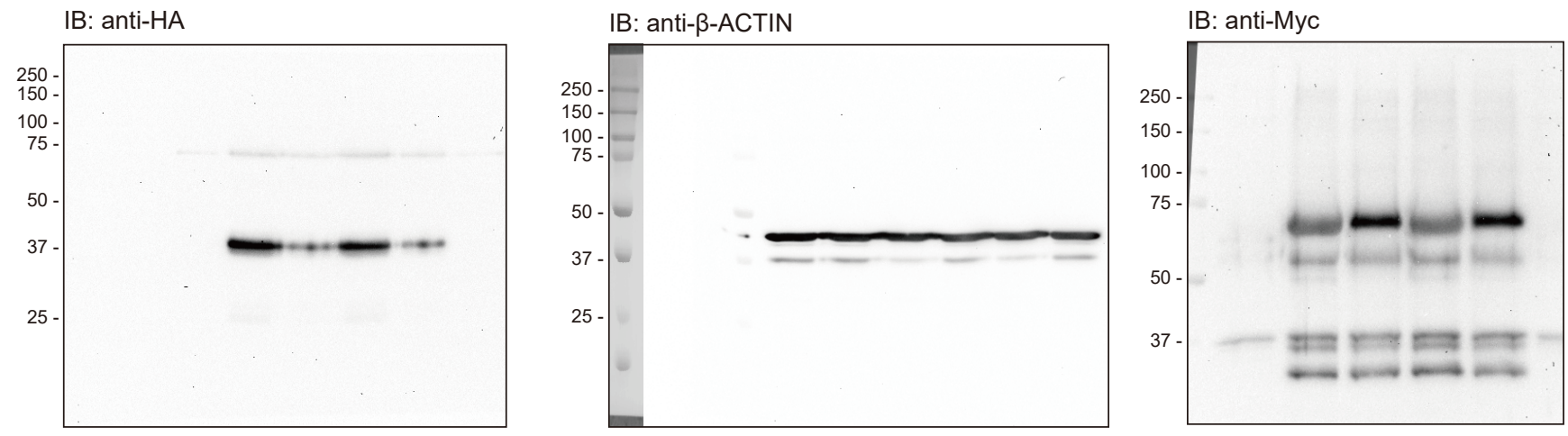
Figure 4D

IB: anti-HA

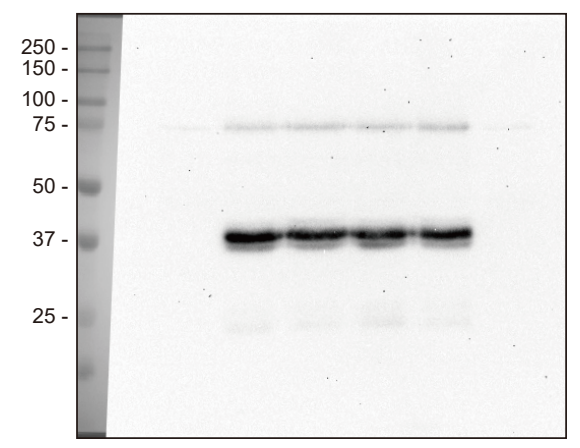

IB: anti-Myc

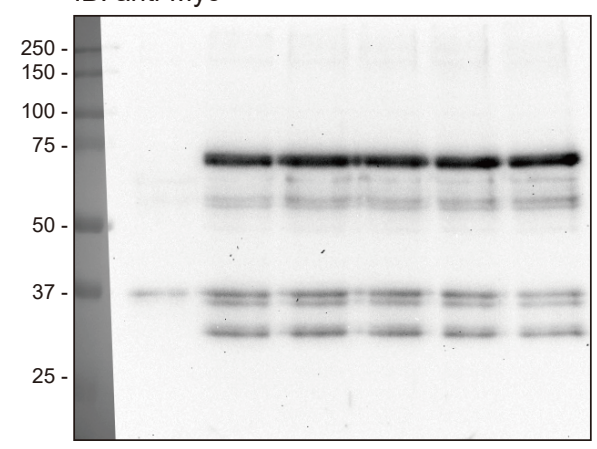

Figure 4E

IB: anti-HA

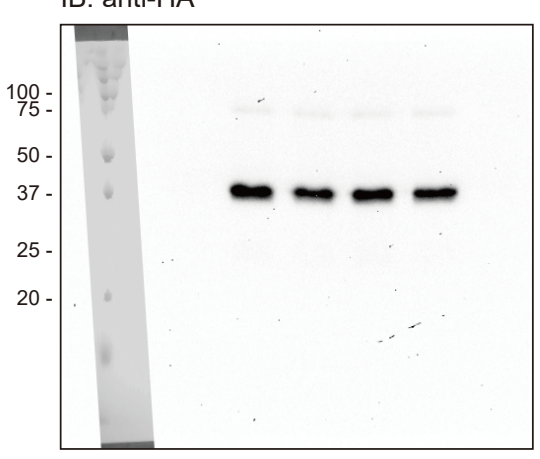

IB: anti-Myc

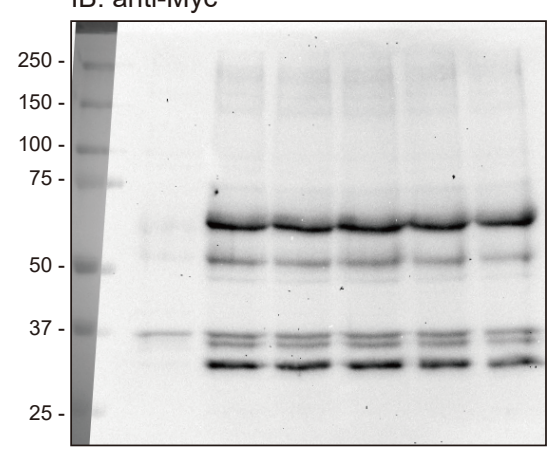

IB: anti- $\beta$-ACTIN
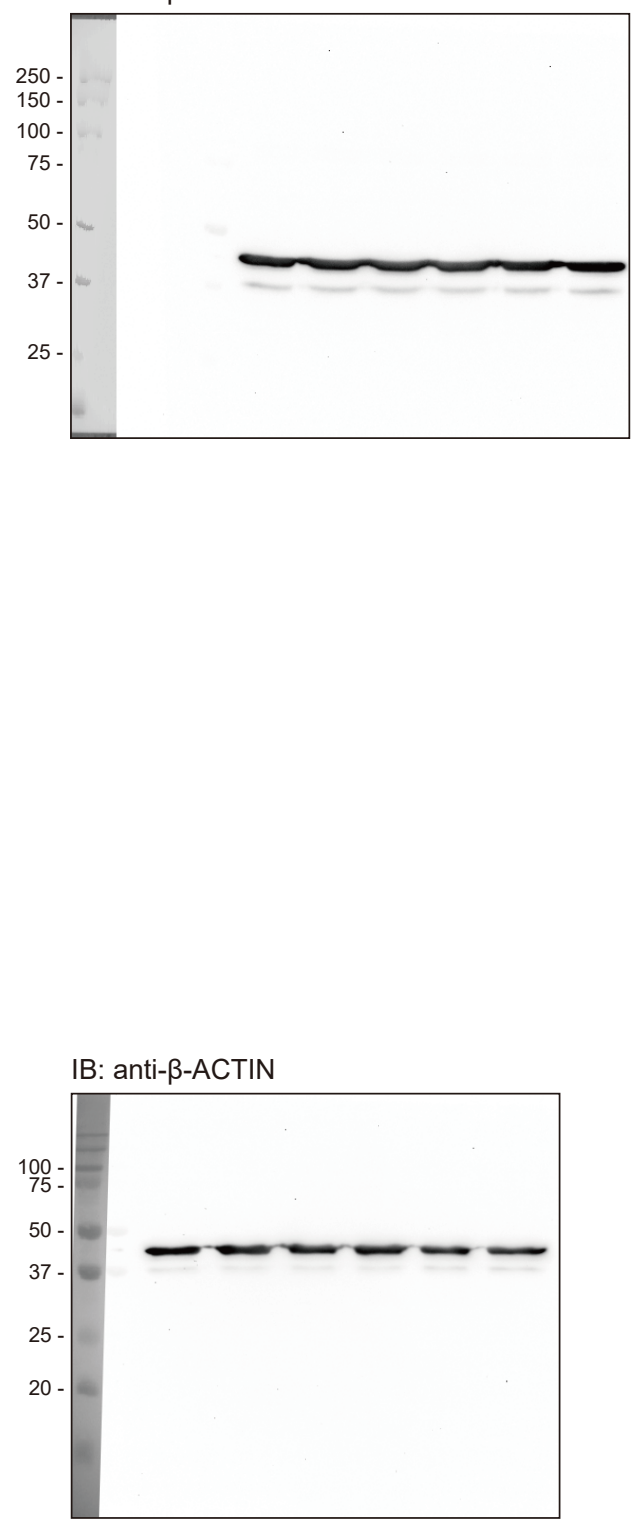
Figure 5B

IB: anti-HA

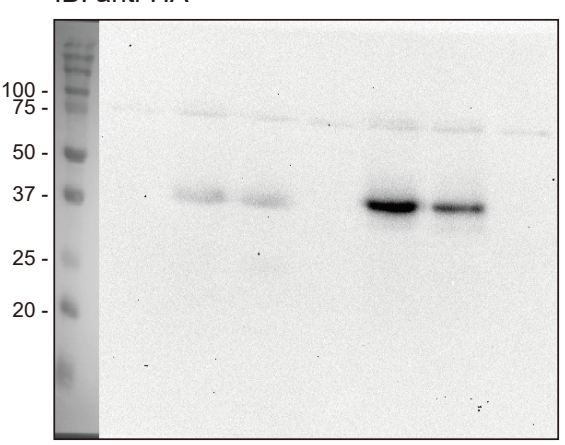

JAM1 $\Delta(1-26)$
IB: anti- $\beta$-ACTIN

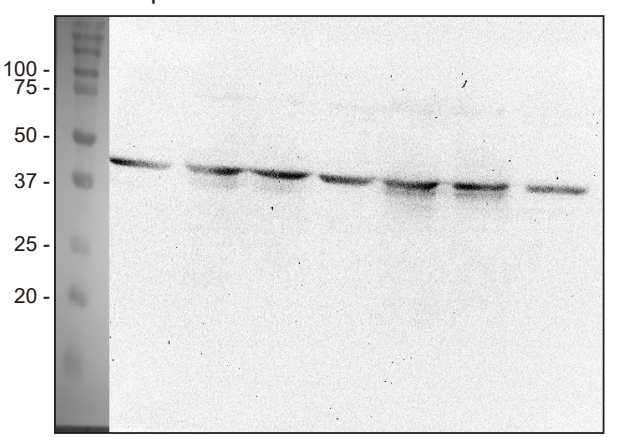

JAM1 $\Delta(1-26)$
IB: anti-HA

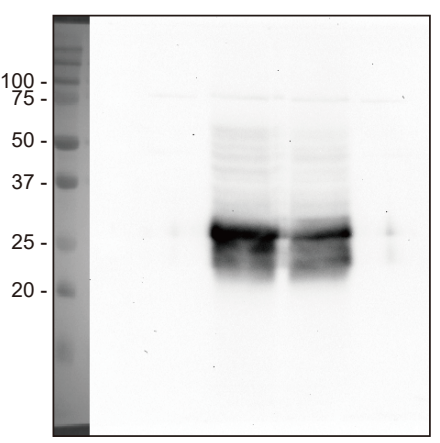

JAM1 $\Delta(1-133)$

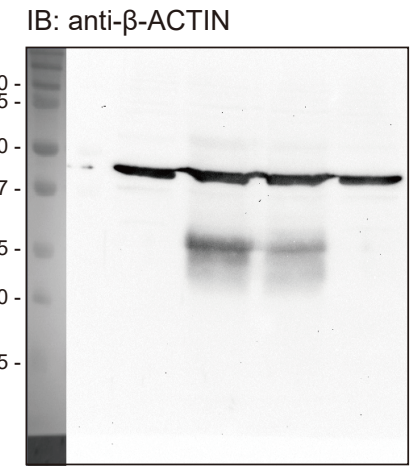

JAM1 $\Delta(1-133)$

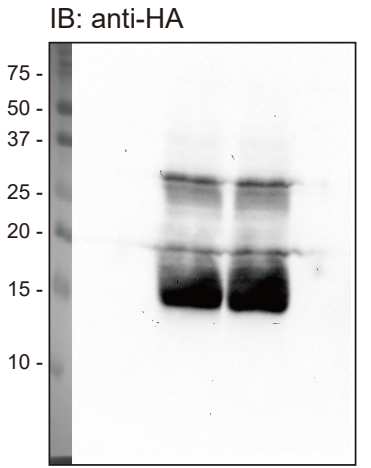

JAM1 $\Delta(1-236)$

IB: anti- $\beta$-ACTIN

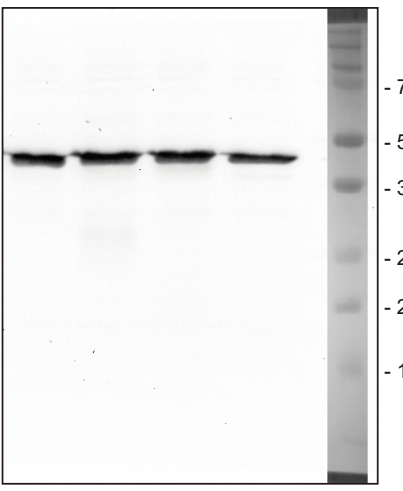

JAM1 WT

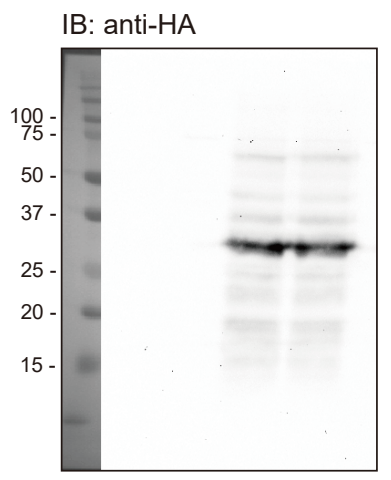

JAM1 $\Delta(237-299)$

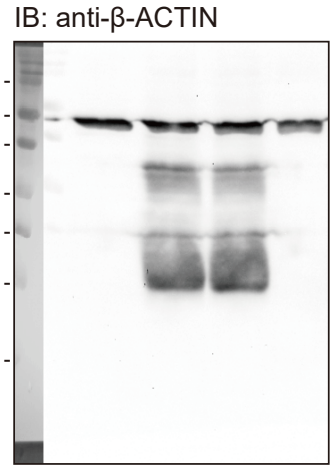

JAM1 $\Delta(1-236)$

IB: anti-HA

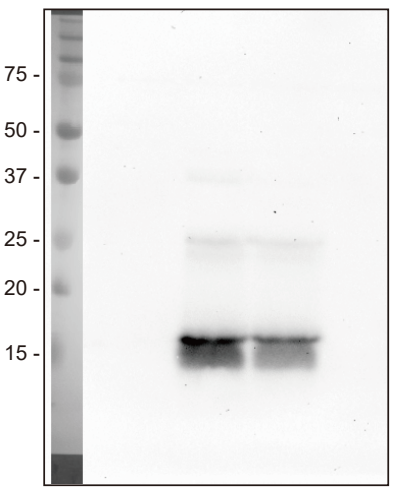

JAM1 $\Delta(1-228)$

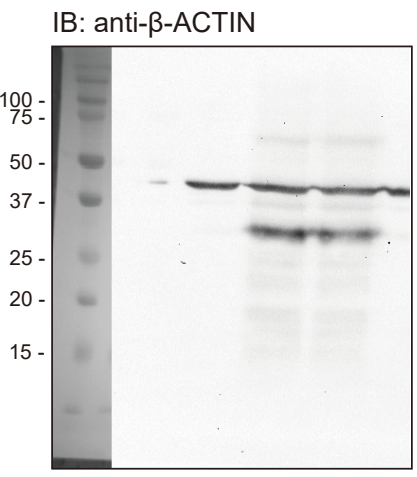

JAM1 $\Delta(237-299)$

IB: anti-HA

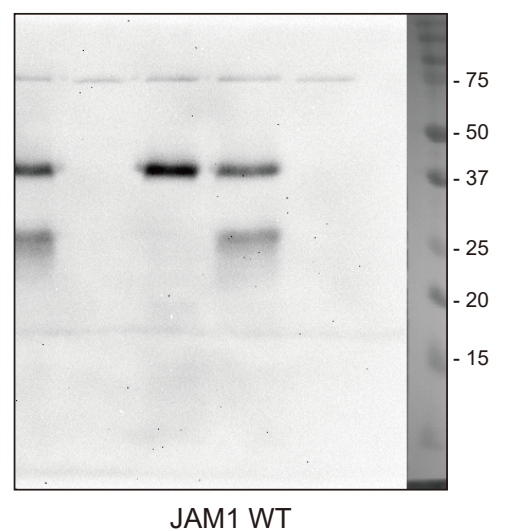

IB: anti- $\beta$-ACTIN

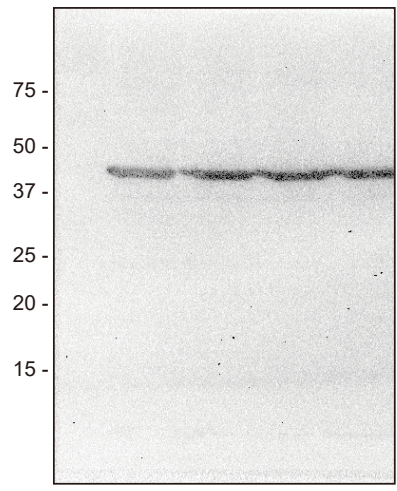

JAM1 $\Delta(1-228)$ 
IB: anti-HA

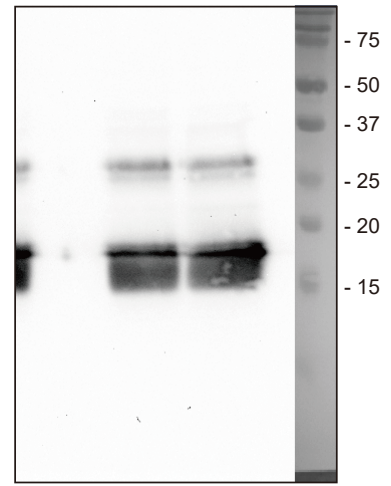

JAM1 $\Delta(1-228) \mathrm{R} 234 \mathrm{H}$

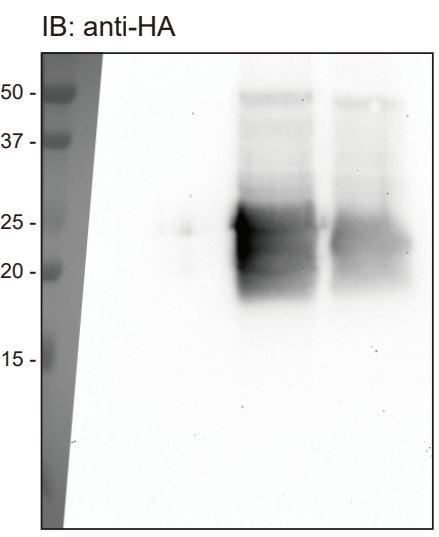

JAM1 $\Delta(1-133) \mathrm{R} 234 \mathrm{H}$

\section{Figure 6B}

IB: anti- $\beta-A C T I N$

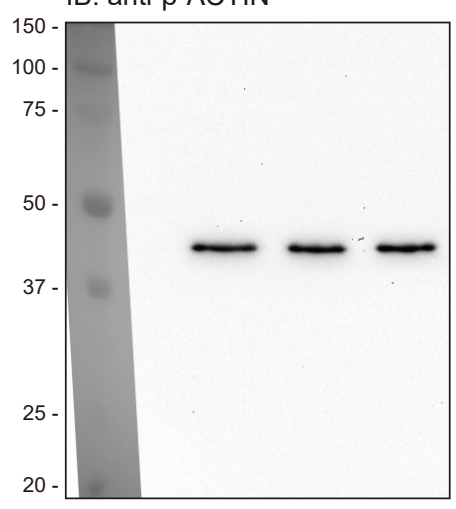

\section{Figure 8H}

IB: anti-JAM1

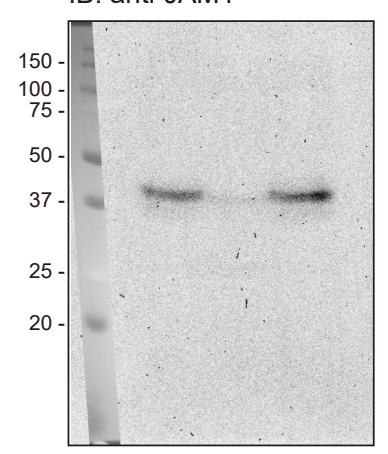

IB: anti- $\beta-A C T I N$

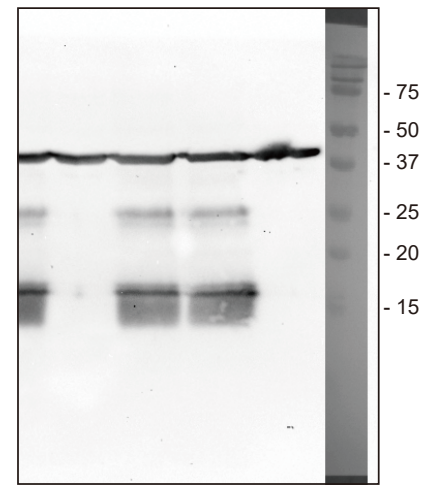

JAM1 $\Delta(1-228) \mathrm{R} 234 \mathrm{H}$

IB: anti- $\beta-A C T I N$

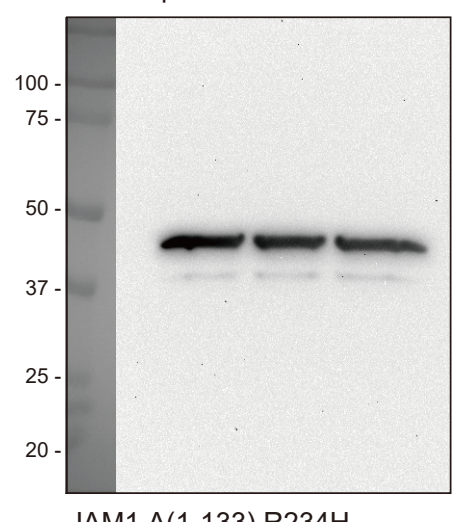

JAM1 $\Delta(1-133) \mathrm{R} 234 \mathrm{H}$
IB: anti-HA

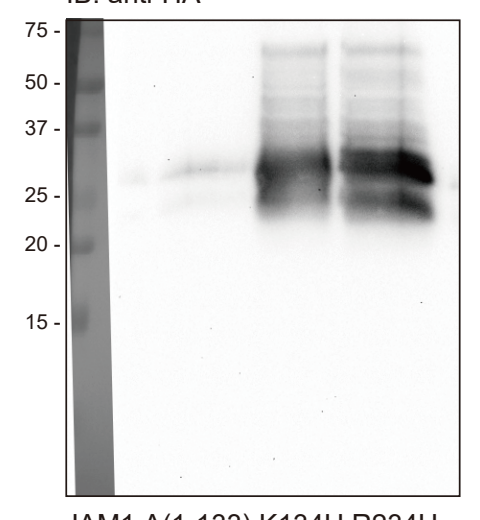

JAM1 $\Delta(1-133) \mathrm{K} 134 \mathrm{H}$ R234H
IB: anti-ß-ACTIN

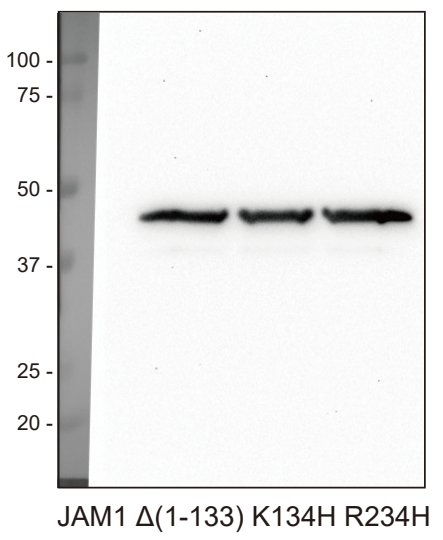

IB: anti-JAM1

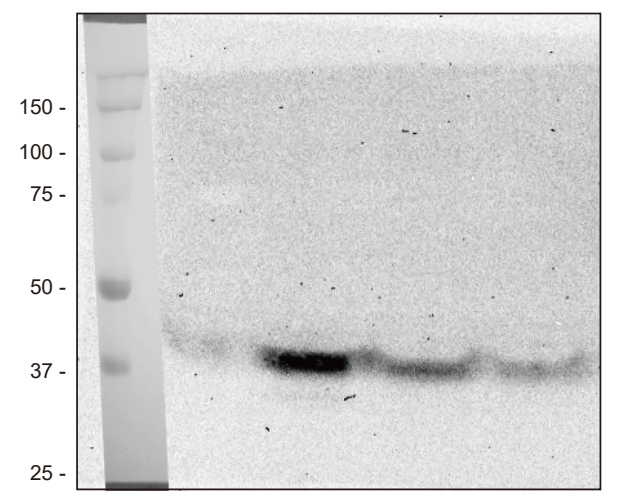

IB: anti- $\beta-A C T I N$

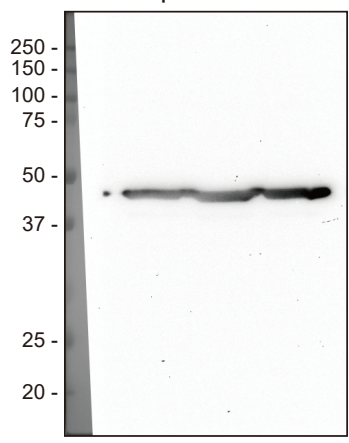




\section{S1 Fig}
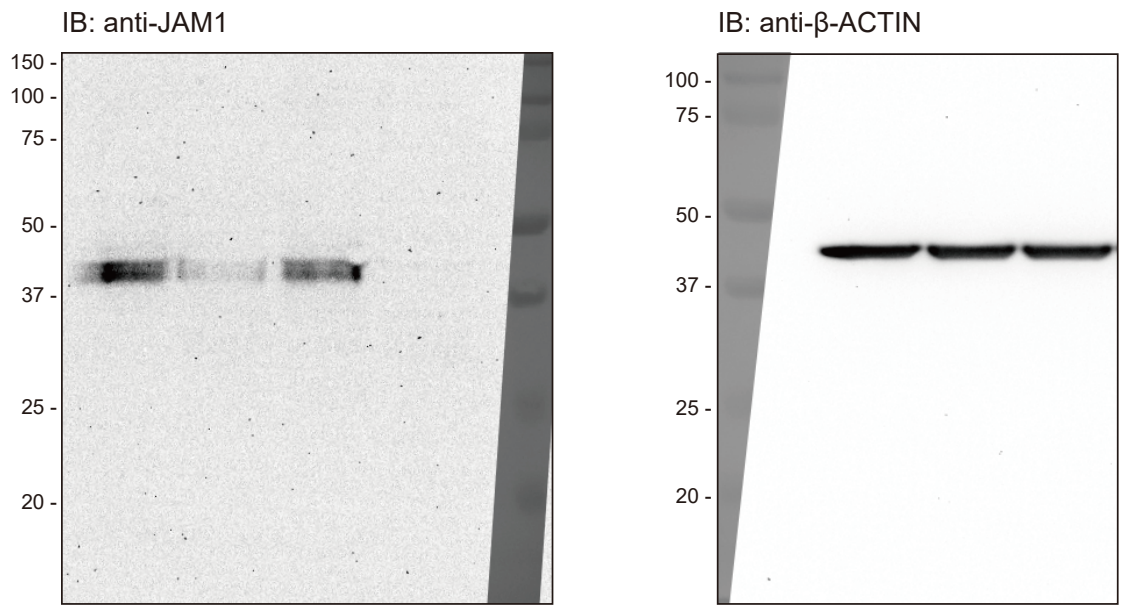

\section{S2 Fig}

IB: anti-Myc

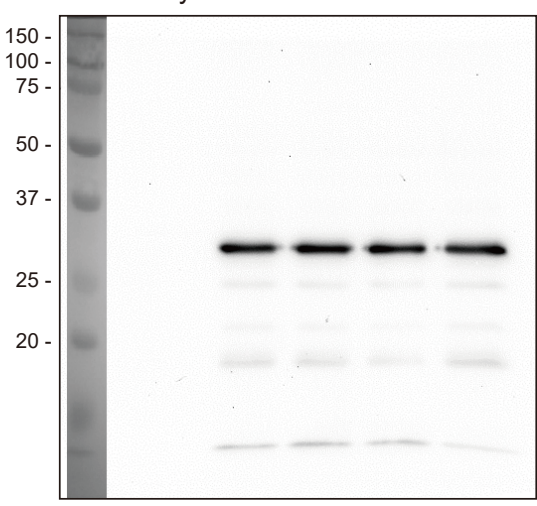

IB: anti- $\beta-A C T I N$

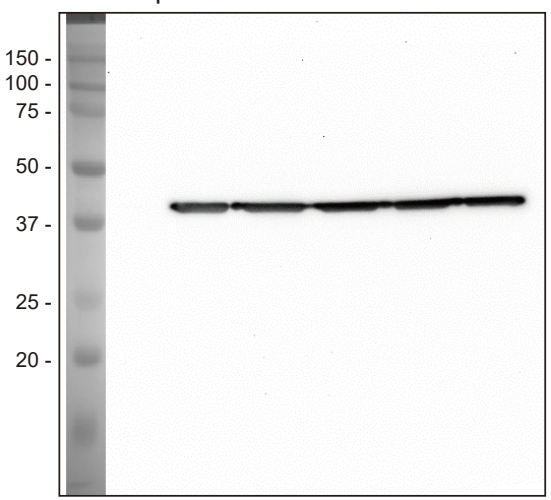

\section{S7 Fig}

IB: anti-Myc

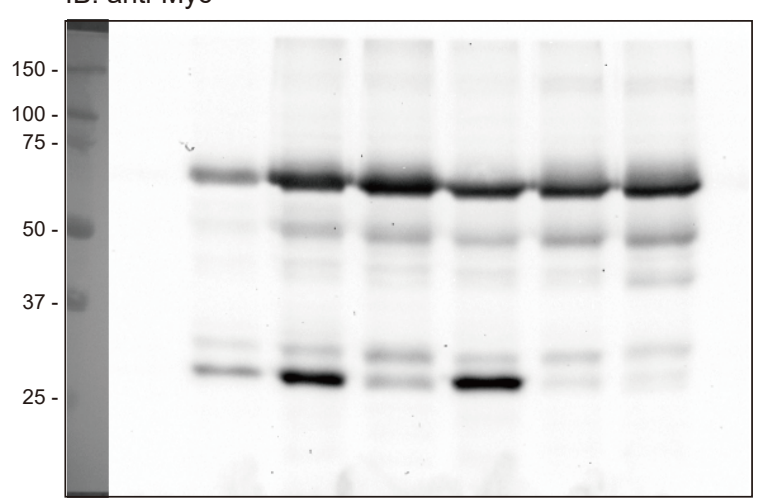

Myc-mCherry-tagged HA-inserted-JAM1
IB: anti-HA

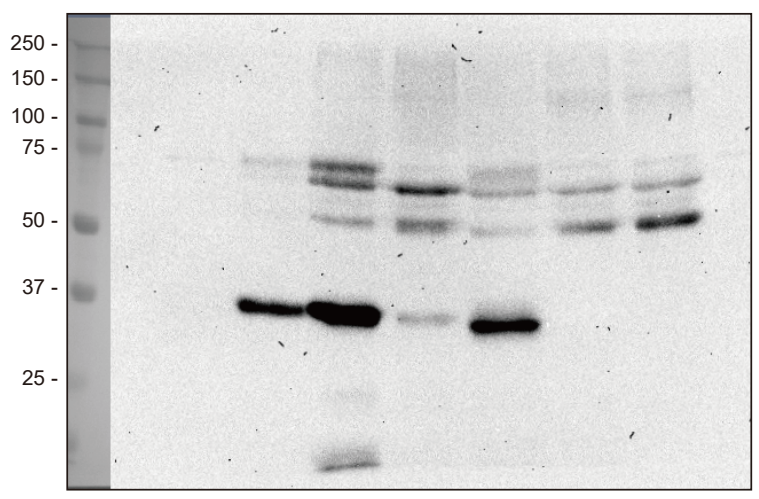

Myc-mCherry-tagged HA-inserted-JAM1 
IB: anti- $\beta-A C T I N$

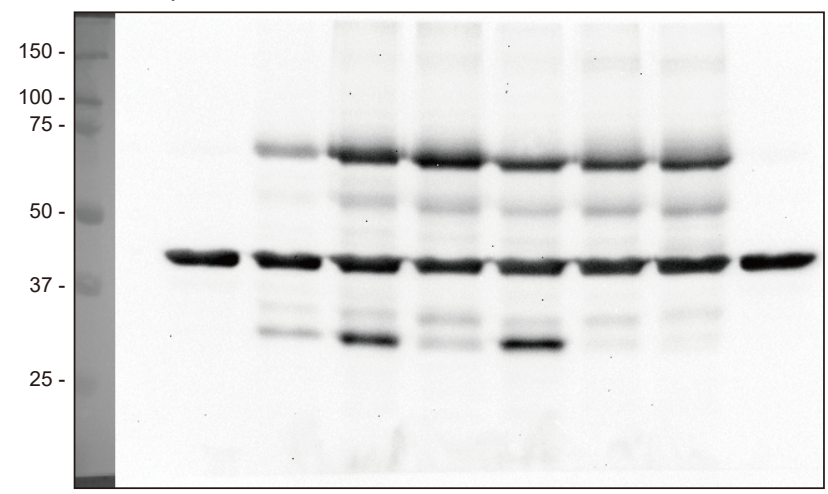

IB: anti-Myc

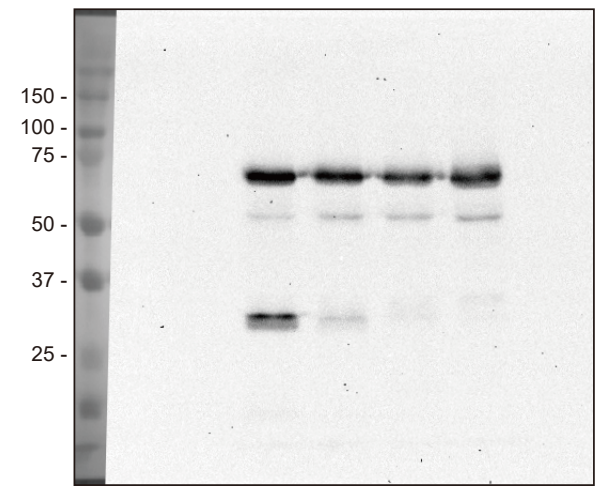

Myc-mCherry-tagged HA-inserted-JAM1

IB: anti- $\beta-A C T I N$

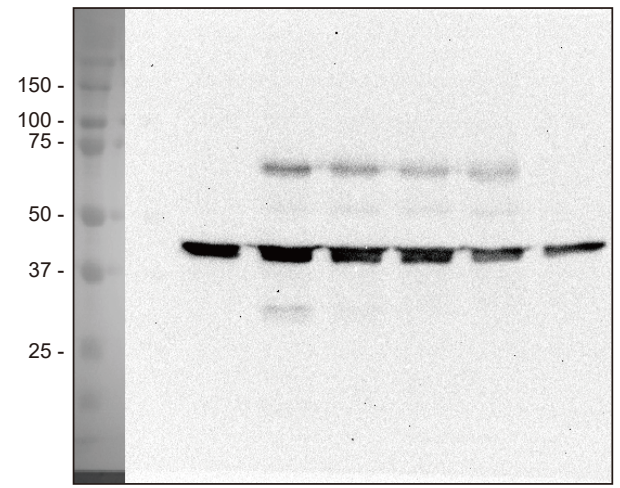

\section{S12 Fig}

IB: anti-Myc

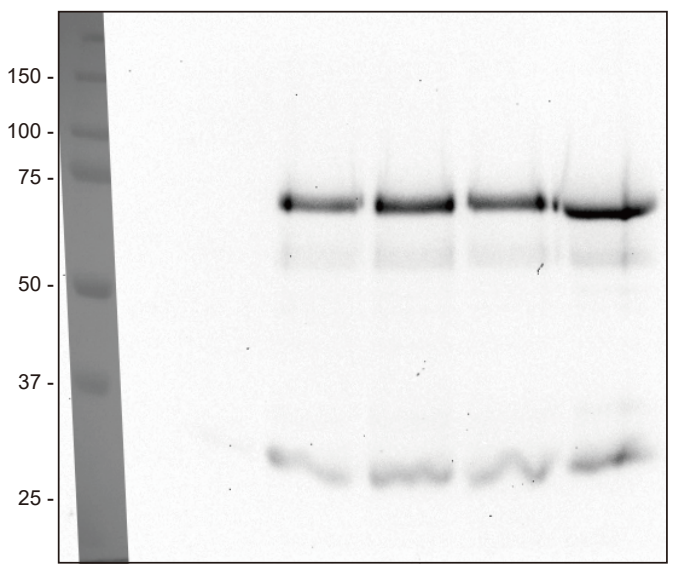

IB: anti-HA

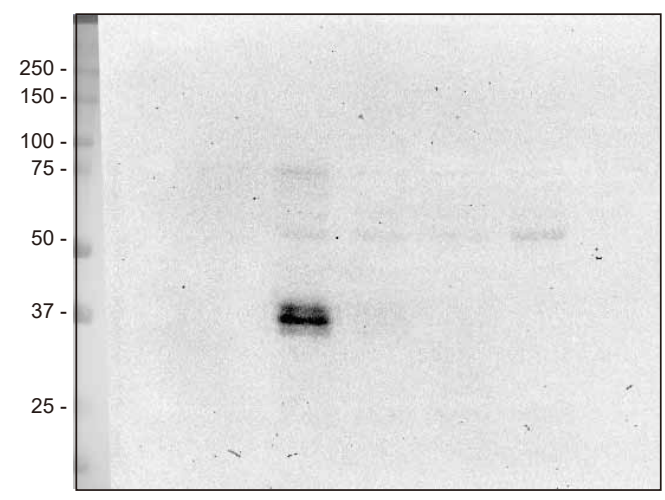

Myc-mCherry-tagged HA-inserted-JAM1
IB: anti-HA

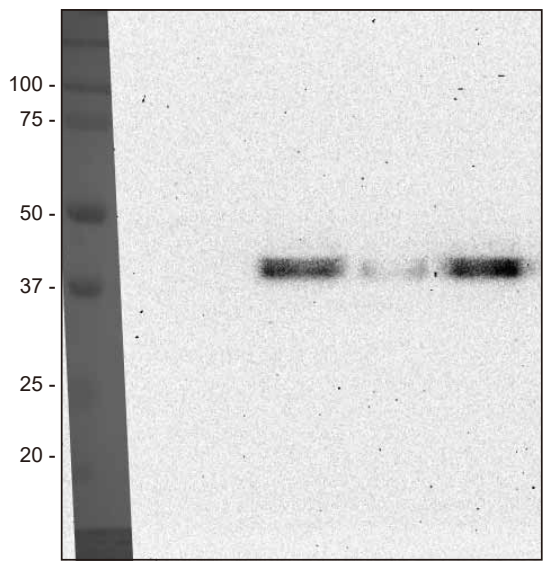

IB: anti- $\beta-A C T I N$

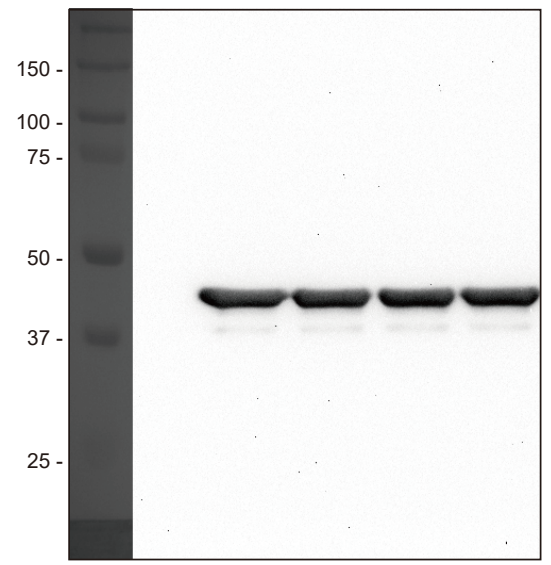

